\title{
Formative evaluation on cultural tailoring breathing awareness meditation smartphone apps to reduce stress and blood pressure
}

\author{
John C. Sieverdes ${ }^{1}$, Zachary W. Adams ${ }^{2}$, Lynne Nemeth ${ }^{1}$, Brenda Brunner-Jackson ${ }^{1}$, Martina Mueller ${ }^{1}$, \\ Ashley Anderson ${ }^{1}$, Sachin Patel ${ }^{1}$, Luke Sox ${ }^{1}$, Frank A. Treiber ${ }^{1,3}$ \\ ${ }^{1}$ Medical University of South Carolina, College of Nursing, Charleston, SC, USA; ${ }^{2}$ Department of Psychiatry, Indiana University, Indianapolis, IN, \\ USA; ${ }^{3}$ Medical University of South Carolina, College of Medicine, Charleston SC, USA \\ Contributions: (I) Conception and design: L Nemeth, B Brunner-Jackson, M Mueller, S Patel, FA Treiber; (II) Administrative support: B Brunner- \\ Jackson, A Anderson; (III) Provision of study materials or patients: B Brunner-Jackson; (IV) Collection and assembly of data: JC Sieverdes, A \\ Anderson, L Sox; (V) Data analysis and interpretation: JC Sieverdes, ZW Adams, L Nemeth, M Mueller, FA Treiber; (VI) Manuscript writing: All \\ authors; (VII) Final approval of manuscript: All authors. \\ Correspondence to: John C. Sieverdes, PhD. Medical University of South Carolina, College of Nursing, Room SN502, 99 Jonathan Lucas St., \\ Charleston, SC 29425-1600, USA. Email: sieverde@musc.edu.
}

Background: Chronic stress is an independent risk factor for essential hypertension (EH), cardiovascular disease (CVD), and is sometimes confronted by mal-adaptive coping behaviors (e.g., stress eating, excessive alcohol consumption, etc.). Pre-essential hypertension (preEH) is the leading predictor of future EH status. Breathing awareness meditation (BAM) can result in clinically beneficial blood pressure (BP) reductions, though face-to-face sessions presents barriers to reach those in need. The purpose of this study was to identify if a culturally tailored approach is needed in the design and preferences between groups of preEH African American and White adults toward using a smartphone BAM app, the Tension Tamer (TT) app.

Methods: TT includes audio delivered BAM instructions, real-time heart rate, feedback graphs and motivational reinforcement text messaging. Questionnaires and two focus groups each of African American and White adults, [n=34, mean age $=43.1$ years, (SD 13.8 years), 44.1\% African American] were conducted to understand stress, EH knowledge, app usage along with feedback from a hands-on demonstration of TT. Grounded theory using NVivo 10 was used to develop themes and combined with the questionnaires in the analysis.

Results: No racial differences were found in the analysis including app use scenarios, preferences, knowledge, technology use or the attitudes and acceptance toward mobile health (mHealth) programs. Reported stress was high for African Americans [PSS-4: mean 6.87 (SD 3.3) versus mean 4.56 (SD 2.6); $\mathrm{P}=0.03$ ]. Four main themes were found: (I) stress was pervasive; (II) coping strategies were both positive and negative; (III) BAM training was easy to incorporated; and (IV) tracking stress responses was useful. Responses suggest that additional personalization of app interfaces may drive ownership and adherence to protocols. Measures and reports of heart rate monitoring while in session were favorably viewed with low issues with confidentiality or trust issues on collected session data.

Conclusions: Results suggest that a culturally tailored approach may be unnecessary in the design of BAM apps. Further investigation is warranted for other racial groups, age ranges, and disease conditions.

Keywords: Essential hypertension (EH); mobile health (mHealth); stress reduction; meditation; grounded theory

Received: 12 July 2017; Accepted: 25 September 2017; Published: 19 October 2017.

doi: $10.21037 /$ mhealth.2017.09.04

View this article at: http://dx.doi.org/10.21037/mhealth.2017.09.04 


\section{Introduction}

Stress is a process in which environmental demands tax or exceed one's adaptive capacity, resulting in physiological changes that may place the individual at risk for disease over time (1-7). Chronic stress may manifest itself in various ways including increased anxiety, depressive symptoms, hostility and poor sleep quality, all which independently predict essential hypertension $(\mathrm{EH})$, cardiovascular disease (CVD), metabolic syndrome and type 2 diabetes (1-6,8). Stress exposure also increases unhealthy coping behaviors such as comfort eating, smoking, excessive alcohol intake, pharmaceutical abuse and increased sedentary activity. These behaviors exacerbate psychological and physiological stress-related symptoms and increase risk for disease (9-15). There is reason to believe health disparities also contribute to stress resulting in differences in trust and engagement in the health care system (16).

Meditation or breathing awareness meditation (BAM) is one of the most studied nonpharmacologic stress reduction strategies. Meta-analytic reviews have shown BAM reduces self-reported stress, anxiety, worry and their associated detrimental physiological effects including elevated blood pressure (BP) $(17,18)$. A number of randomized clinical trials have been conducted using BAM and have indicated high acceptability, usability, and beneficial effects on stress levels, physiological functioning, and reduced casual and ambulatory BP $(17,18)$. Our prior research has also found BAM highly acceptable, easy to learn and has resulted in reductions of stress, hostility, resting and ambulatory $\mathrm{BP}$ and improved sodium handling (19-23). Although BAM programs have been found effective, they are usually taught face-to-face, requiring individual or group classes and transportation, which may represent financial or timeassociated barriers to those with the most need. Innovative solutions are needed to increase accessibility of this effective intervention.

$\mathrm{BAM}$ research also has lacked efficacious objective measure of adherence to BAM practice sessions outside of the classroom or clinic, effecting the true reported dosage conditions (17,24-26). The actual amount of BAM dosage is unknown in many studies and may partially contribute to the substantial heterogeneity in individuals' BP changes observed within and across trials. With the ubiquity of smartphones and continued growth in mobile health (mHealth) apps, a proliferation of stress reduction apps are now available on app stores. These promote various forms and tracking of BAM or meditation practices involving diaphragmatic breathing (27). Although apps help overcome some logistical barriers to implementing BAM for stress reduction, adherence monitoring and maximizing program uptake remains a problem.

Culturally tailored approaches have been an important element to foster behavioral change program design leading to greater adherence, program acceptance and adoption. A large body of evidence supports this approach to tailor programs for specific communities, ethnicities, or cultures and warrants investigation in the area of BAM (28-30). Such work is particularly important given racial and ethnic disparities in health and health care across a wide range of disease categories influenced by stress (16). Several mHealth programs have incorporated this approach and have reported favorable effects in several areas from diabetes to HIV care (31,32). Therefore, although there are added costs associated with adopting an mHealth design and development process that involves cultural tailoring, it appears to be promising in improving user engagement and outcomes.

This report presents the next step in the iterative design process of a BAM app called Tension Tamer (TT) and provides a case example to illustrating if culturally tailoring BAM apps is warranted. Responses from White and African American users were compared with TT app interactive preferences, presentation formats, training on the use of apps, use case scenarios, trust about privacy, general knowledge about EH and identifying cultural differences and similarities in prevalent stress associated behaviors to inform design characteristics and refinements. We further explored and examined cultural attitudes toward the use of mHealth technology for prevention and management of stress as a coping method. We hypothesized that different thematic findings through this qualitative investigation would be evident to warrant culturally-sensitive tailoring in future TT app refinement and design of other BAM or mHealth apps.

\section{Methods}

Similar to our approach in our other recent mHealth development programs, a qualitative approach consisting of four focus groups and a battery of questionnaires were used (33-35). Two focus groups of White and two groups of African American participants were completed to investigate differences in needs, preferences, perceptions of $\mathrm{EH}$ and stress, and their use of smartphone apps to address health concerns. A hands-on demonstration of the TT app 
was used to collect feedback on the usage and perception of BAM apps. Data was collected both quantitatively and qualitatively to define preferences toward using a BAM app and racial or culturally tailored themes. The Consolidated Criteria for Reporting Qualitative Studies (COREQ) 32-Item Checklist was followed when reporting the findings of this study (36).

\section{Background of TT}

Over the past 6 years, we have incorporated a patientcentered, behavioral theory-guided, iterative design process to deliver BAM through TT on Android and iOS platforms (37). Very little evidence is available on the efficacy of other meditation/BAM apps in developing effective habits and mindfulness, and none have documented outcomes on BP besides TT (38). Initially, pre-essential hypertension (preEH) and $\mathrm{EH}$ youth and adults guided the structure and refinement of early TT app content and presentation through focus groups [e.g., TT app consists of a brief overview communicated via cartoon and text, FAQ, audio and text based BAM instructions, heart rate (HR) feedback graphs, etc.]. A proprietary software program was developed to enable validated realtime HR acquisition during TT sessions using the built-in LED and video camera as a reflective photoplethysmograph $(39,40)$. We have observed promising results from two 3 -month smartphone delivered TT trials $(37,41,42)$, where TT was found to be highly acceptable and intuitive in its use. In addition, clinically meaningful reductions in resting $\mathrm{BP}$, ambulatory BP and reductions in SNS activation (i.e., reduced morning awakening curve of salivary alpha amylase) have been observed in preEH White and African American adults outlining a biological mechanistic pathway for BAM and resultant stress reduction $(15,41,42)$.

\section{Participants}

The target audience of the TT app were adults with preEH due to the established risk trajectory of preEHs developing EH. Longitudinal studies have shown that the 4-year incidence of developing $\mathrm{EH}$ in higher stratum preEH categories (i.e., SBP 130-139 mmHg and/or DBP 85-89 $\mathrm{mmHg}$ ) is $43 \%$ compared to $20 \%$ in lower preEH stratums (SBP 120-129 mmHg and or DBP $80-84 \mathrm{mmHg}$ ) (43). Study participants were identified from a list of adult patients with preEH from a large family medicine practice through flyers posted at an academic medical center and at a college campus in a southeastern coastal city in the USA. Inclusion criteria consisted of preEH status, both genders and ownership or non-ownership of a smartphone as future clinical trials will offer study smartphones. Two focus groups of White and two focus groups of African Americans were recruited. Participants who responded to recruitment materials without a physician diagnosis of preEH were prescreened using standard BP screening procedures in a quiet conference room where $3 \mathrm{BP}$ readings were taken $3 \mathrm{~min}$ apart after a 5 -min seated rest. The last two measures were averaged to assess if $\mathrm{BP}$ were in preEH ranges. Enrolled participants provided written informed consent prior to the focus group visit. All study procedures were approved by the academic center's institutional review board prior to the start of research activities.

\section{Procedure}

Four focus group sessions were conducted on separate days over a 1-month period. Sessions were conducted in a large conference room at an academic medical center, facilitated by two members of the research team (PhD and $\mathrm{MPH}$ level researchers) and were audio recorded with field notes capturing nonverbal responses. Two sets of questionnaires were given to participants. The first set of questionnaires was given prior to the focus groups and consisted of demographics, mobile/smartphone use patterns, stress levels and hypertension knowledge. The second set was given at the end of the sessions and is described later. After an introduction, the facilitators questioned the participants about their knowledge of $\mathrm{EH}$ and potential sources and levels of stress using a semi-structured scripted interview format. Afterward, a brief education session (4-6 min) about the beneficial impact of BAM programs on perceived stress levels and BP control was given. Next, each participant was provided an iPhone (Model 4S, Apple, Inc., Cupertino, CA, USA) with a fully operational version of the TT app for demonstration purposes and completed a hands-on demonstration. The guided demonstration included an appdirected introduction tutorial (i.e., a quick-start tutorial and a voice-guided BAM session). After the demonstration, the participants used the TT app for 15-20 min to explore its features. Observations were taken about the groups' immediate reactions to the app during this time where participants were asked to voice out loud what they were thinking. Participants were asked about their perceptions and reactions to TT's look, feel and functionality to gather input for further refinements of the TT app as well as 
their needs and experience using other mHealth apps. A summary of the discussions and suggestions for refinements were voiced by the facilitators to confirm groups' responses to correct any misinterpretations.

The second set of questionnaires followed at the end of the session and focused on participants' attitudes toward mHealth technology for disease prevention and to quantitatively assess their perceptions of the TT app. Participants were each compensated $\$ 50$ for their time. Focus group sessions lasted between 60 and $75 \mathrm{~min}$.

\section{Questionnaire measures: pre-focus group}

Participants completed surveys on demographics (7 questions: i.e., age, marital status, income, education level, employment status, etc.), their experience with technology and use of cell phones features (14 questions: yes/no and 4-point Likert scales). Participants also completed the 4-question Perceived Stress Scale-4 (PSS-4) (4 questions; 5-point Likert scales), a knowledge of EH scale [13 questions; 3-4 answer multiple choice; 1 correct answer per question (EH diagnosis ranges, effects of $\mathrm{EH}$ ), etc.] and attitudes toward mHealth for use in health promotion and disease (8 questions: yes/no and 5-point Likert scales responses). The PSS-4 stress scale is well established (PSS-4; alpha reliability $=0.60)(44)$ and has been used in a variety of clinical settings. The scales on the use of smartphones and attitudes toward use in health promotion and disease prevention has shown high internal consistency in previous studies $($ Cronbach's alpha $=0.92)(33,34)$.

\section{Questionnaire measures: post-focus group}

At the completion of the focus groups, additional questionnaires included assessing perceived acceptability of the TT app, belief the TT app would be useful in lowering $\mathrm{BP}$ and stress and ease of use were administered (18 questions; 5-point Likert scales).

\section{Focus group script development and content}

The focus group script was developed by the authors using the guidelines from How to Design Survey Studies: The Survey Kit (45). The technology acceptance model (46) guided the use case and technology interface questions along with discussions among the research team to target themes of usability, reactions to app-based bio-behavior feedbacks, adherence, app reactivity, locations of use, and educational delivery modality. Questions were created using constructs outlined by the self-determination theory (47) and technology acceptance model (46) to determine underlying motivations and develop user interaction, acceptability, and perceptions of the TT app. Questions were vetted by a team of clinical psychologists, clinical researchers and qualitative experts with the intention to identify different use cases, motivations, interface perceptions, and graphic user interface (GUI).

The focus group questions targeted several topics. The first topic involved the level and type of stress in life, coping behaviors and stress's potential ramifications on BP levels (i.e., do you think you have stress? How do you currently try to reduce your stress? etc.). Following the subsequent EH and BAM education session, participants were prompted regarding their impressions during the hands-on demonstration of the TT app: the system in general, smartphone BAM/meditation training, pulse detection of the camera, concerns about the app tracking their information, reminders to use the app, daily frequency of BAM, and encouragement messages and information the TT app requested. Discussions were expanded to encompass mHealth apps in general with an emphasis on BAM apps. Usefulness, ease of use, and improvements were qualitatively assessed for each topic area describe above. Final discussions involved whether participants held the belief that using a BAM app could help improve health or $\mathrm{BP}$ by reducing stress levels, whether they had concerns with the confidentiality, where and how much BAM users would perform, technical skills required to successfully integrate the app in their lives, concerns on the lack of personal contact with the program, and feedback on the HR summary report.

\section{Statistical analysis}

Questionnaire responses were analyzed using IBM SPSS Statistics v20 (SPSS IBM, New York, USA). Responses were stratified by racial groups using means, standard deviations (SD) and percentages. Significance between groups were tested using independent $\mathrm{t}$-tests for continuous data and chisquare tests for categorical data using a $\mathrm{P}$ value $\leq 0.05$. The focus group audio recordings were sent for transcription using HIPAA compliant file transfer procedures. Transcripts were imported into NVivo 10.0 (QSR International Pty, Doncaster, Victoria, Australia) for qualitative analysis to define themes for further evaluation (48). Inductive content analysis was used to distill text into fewer content-related 
Table 1 Characteristics of participants in focus groups

\begin{tabular}{|c|c|}
\hline Variable & Participants $(n=34)$ \\
\hline Age (years) & $43.1 \pm 13.8$ \\
\hline \multicolumn{2}{|l|}{ Race, n (\%) } \\
\hline White & $18(52.9)$ \\
\hline Black & $15(44.1)$ \\
\hline Other & $1(2.9)$ \\
\hline \multicolumn{2}{|l|}{ Gender, n (\%) } \\
\hline Male & $14(41.2)$ \\
\hline Female & $20(58.8)$ \\
\hline \multicolumn{2}{|l|}{ Marital status, n (\%) } \\
\hline Single & $11(32.4)$ \\
\hline Married & $17(50.0)$ \\
\hline Divorced & $5(14.7)$ \\
\hline Living with significant other & $1(2.9)$ \\
\hline \multicolumn{2}{|l|}{ Education, n (\%) } \\
\hline High school or less & $3(8.8)$ \\
\hline At least some college & $31(91.2)$ \\
\hline \multicolumn{2}{|l|}{ Income, n (\%) } \\
\hline$<\$ 15 K$ & $9(26.5)$ \\
\hline$\$ 15-29.9 K$ & $3(8.8)$ \\
\hline$\$ 30-49.9 \mathrm{~K}$ & $11(32.4)$ \\
\hline$\$ 50-75 \mathrm{~K}$ & $3(8.8)$ \\
\hline$>\$ 75 \mathrm{~K}$ & $5(14.7)$ \\
\hline Not reported & $3(8.8)$ \\
\hline \multicolumn{2}{|l|}{ Employment, n (\%) } \\
\hline Full-time & $23(67.7)$ \\
\hline Part-time & $5(14.7)$ \\
\hline Unemployed & $4(11.8)$ \\
\hline Disabled & $2(5.9)$ \\
\hline
\end{tabular}

$P$ values $\geq 0.45$ for all categories between White and African American groups.

categories or themes, where words and phrases in a category share similar meaning (49). Each transcript was openly coded by a primary qualitative analyst using NVivo software in a line-by-line inductive fashion to identify key segments of text found in the transcripts. This process of open coding each document avoided the use of preconceived categories and resulted in specifics from the focus groups that were further refined into key themes regarding the potential use of the TT app and differences between racial groups. Through the process of developing new codes up to conclusion of the analysis, we were able to reach saturation of themes where no new concepts were found indicating that additional focus groups were not required. The specific coding structure and examples of the predominant codes found within the coding summary were reviewed by two of the authors. This approach integrated data from nonverbal cues and NVivo transcript analysis using a process of immersion and crystallization that confirmed the themes from all data sources (50). A comparison and contrast between the qualitative and quantitative results were then performed.

\section{Results \\ Demographics}

Descriptive demographic characteristics of the participants $[\mathrm{n}=34$, mean age $=43.1$ years (SD 13.8 years), $44.1 \%$ African American] are reported in Table 1. Group characteristics revealed that most had attended one or more years of college or a technical trade school (91\%), half were married $(50 \%)$ and most were working full or part time (82.4\%). There were no significant differences between racial groups for education, employment, income, or the other measured demographic characteristics (all $\mathrm{P}$ values $>0.41$ ). The PSS4 found that that African American groups had significantly higher stress than White groups [mean 6.87 (SD 3.3) versus mean 4.56 ( $\mathrm{SD} 2.6) ; \mathrm{P}=0.03]$.

\section{Overall use of mobile phone and smartphone technologies}

Use of mobile phone and smartphone technologies were found to be homogeneous among Whites and African Americans (all $\mathrm{P}$ values $>0.13$; Table 2). Ninety-seven percent of the cohort had a mobile or smartphone [the other $3 \%(n=1)$ reported sharing a spouse's mobile phone] with $75.8 \%$ owning a smartphone. Most participants (90.6\%) noted they lived with someone that could assist them with smartphone or computer tablet technology if they required guidance using apps. Text messaging access in households was $100 \%$ with $87.9 \%$ reporting having unlimited talk time. Mobile phone app downloading was widespread as was using email ( $75 \%$ reported having email). Tablet use was highly prevalent (78.1\% daily usage) for the 
Table 2 Overall use of mobile phone and smartphone technologies

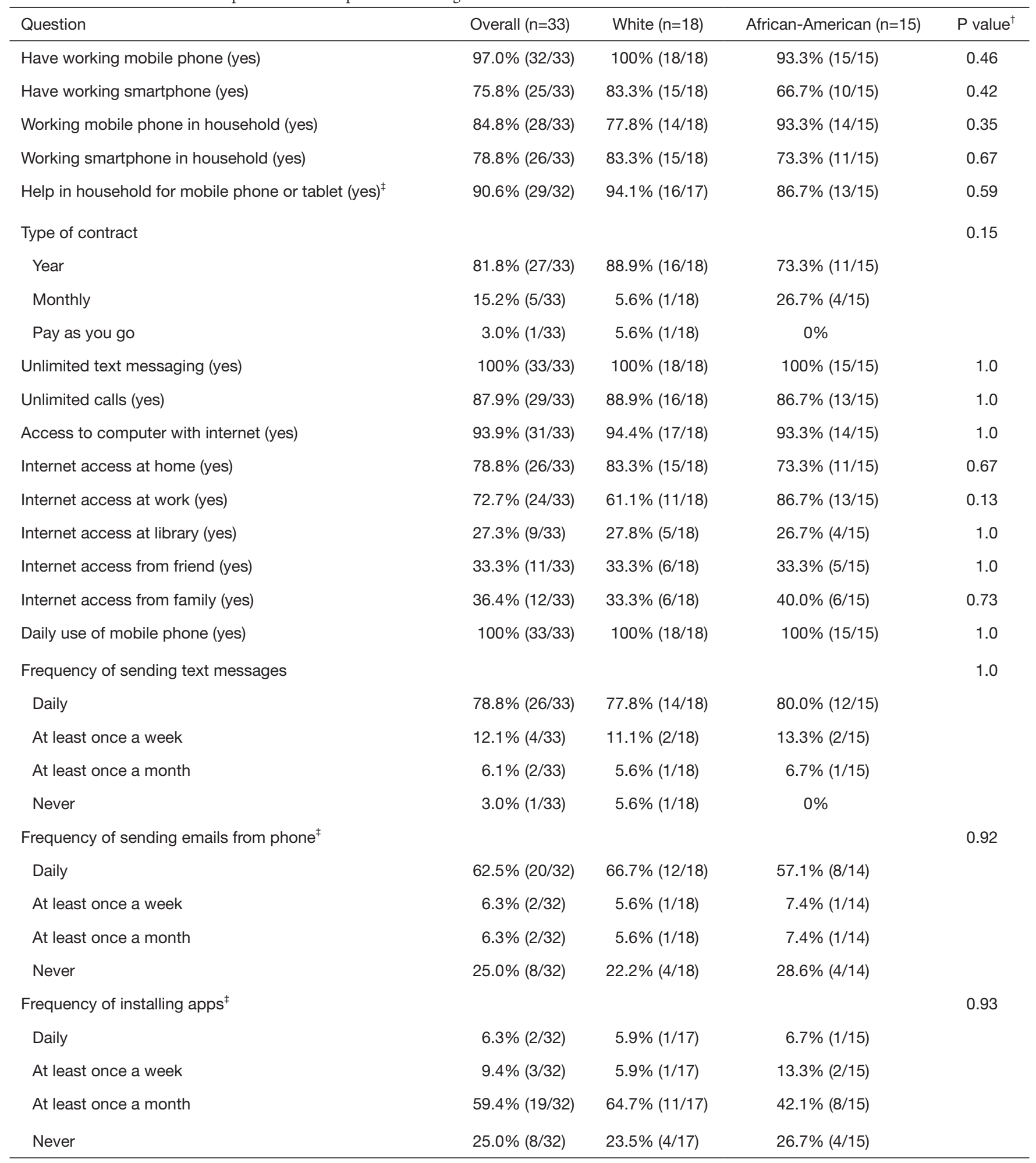

Table 2 (continued) 
Table 2 (continued)

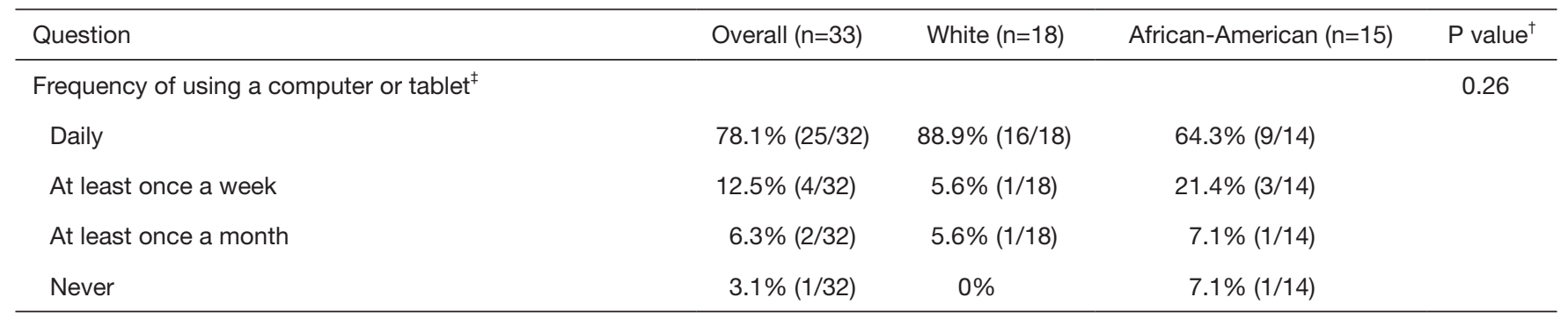

${ }^{\dagger}$, Fisher's exact test; ${ }^{\ddagger}$, one person did not provide answer; P values analyzed using comparison of "daily" versus "less than daily or never".

groups with only one person (3.1\%) who had never used a computer tablet.

\section{Hypertension knowledge}

Hypertension knowledge indicated low levels of understanding on which $\mathrm{BP}$ ranges were considered normal, as well as the resultant etiology on the risk of prehypertension (e.g., that preEH eventually leads to hypertension). Most participants did understand that hypertension leads to strokes or heart attacks and proper treatment involves daily medication regimes, losing weight and reducing salt intake. Scores from the 13-item Hypertension knowledge survey averaged 8.39 out of possible 13 correct answers with no statistical differences found between African American [mean 8.67 (SD 2.1)] and White groups [mean 8.17 (SD 1.5)] $(\mathrm{P}=0.35)$.

\section{Attitudes toward mHealth}

Post-focus group survey responses found no differences between racial groups on using smartphone-based telehealth services (see Table 3). Most (65.6\%) had never heard of telehealth or mHealth prior to the focus groups. The questionnaires prompted the participants to rate their degree of acceptability toward mHealth across two domains: use of mHealth tools overall, and use of the TT app specifically. Ninety-four percent of participants strongly agreed or agreed that they would use mHealth services for prevention or management of chronic diseases if the smartphone and app were free and $87.9 \%$ strongly agreed or agreed to use if only the app were free. A majority $(84.8 \%)$ also felt comfortable if a healthcare provider monitored their health information (i.e., HR responses). Although most (97.0\% strongly agreed/agreed) that TT could lower stress, many did not make the connection between lowering stress results in lower BPs (only $66.7 \%$ responded strongly agreed/agreed).

\section{Qualitative findings}

Primary themes were identified through the qualitative investigation and resulted in no differences in primary themes between White and African Americans groups. Therefore, data were combined and findings were reported as similarities between groups. Four main themes were found in all focus groups. These included: (I) stress was pervasive; (II) coping strategies were both positive and negative; (III) BAM training was easy to incorporate; and (IV) tracking stress responses with a metric was useful. The following describes the results of these themes in detail with themes supported by exemplar quotes in Table 4.

\section{Theme I: stress was pervasive}

The participants noted many potential causes of stress that led to stressful life situations and the resultant effects it held on their health. Some participants had one or two things that they focused on that gave them increased stress while others described multiple hardships or stressful events that kept their perceived stress elevated.

\section{(i) Sub-theme: types of stress}

These reasons ranged from financial, children and family related issues, medical, work-related stress to general life chaos. Importantly, most had multiple causes of stress rather than one particular item and was usually described in a listed format. Non-verbal responses indicated that caring for others with medical conditions and financial strain causing the subject to work multiple jobs may be stronger causes of stress. 
Table 3 Attitudes and acceptance toward mobile health and telehealth programs

\begin{tabular}{|c|c|c|c|c|}
\hline Question & Overall $(n=33)$ & White $(n=18)$ & African-American $(n=15)$ & $P$ value \\
\hline Have heard of telehealth or mobile health before (yes) ${ }^{\ddagger}$ & $34.4 \%(11 / 32)$ & $38.9 \%(7 / 18)$ & $28.6 \%(4 / 15)$ & $0.71^{\dagger}$ \\
\hline Would use free app and smart phone if provided & & & & 0.56 \\
\hline Strongly agree & $45.5 \%(15 / 33)$ & $38.9 \%(7 / 18)$ & $53.3 \%(8 / 15)$ & \\
\hline Agree & $48.5 \%(16 / 33)$ & $50.0 \%(9 / 18)$ & $46.7 \%(7 / 15)$ & \\
\hline Strongly disagree & $3.0 \%(1 / 33)$ & $5.6 \%(1 / 18)$ & $0 \%$ & \\
\hline Would use app at no cost & & & & 0.69 \\
\hline Strongly agree & $51.5 \%(17 / 33)$ & $44.4 \%(8 / 18)$ & $60.0 \%(9 / 15)$ & \\
\hline Feel comfortable provider monitoring health information & & & & 0.14 \\
\hline Strongly agree & $39.4 \%(13 / 33)$ & $38.9 \%(7 / 18)$ & $40.0 \%(6 / 15)$ & \\
\hline Agree & $45.5 \%(15 / 33)$ & $50.0 \%(9 / 18)$ & $40.0 \%(6 / 15)$ & \\
\hline Neutral & $9.1 \%(3 / 33)$ & $0 \%$ & $9.1 \%(3 / 15)$ & \\
\hline Strongly disagree & $6.1 \%(2 / 33)$ & $11.1 \%(2 / 18)$ & $0 \%$ & \\
\hline Feel comfortable using cell phone & & & & 0.64 \\
\hline Strongly agree & $72.7 \%(24 / 33)$ & $72.2 \%(13 / 18)$ & $73.3 \%(11 / 15)$ & \\
\hline Agree & $24.2 \%(8 / 33)$ & $22.2 \%(4 / 18)$ & $26.7 \%(4 / 15)$ & \\
\hline Believe TT app will help lower blood pressure ${ }^{\ddagger}$ & & & & 0.22 \\
\hline Strongly agree & $46.9 \%(15 / 32)$ & $38.9 \%(7 / 18)$ & $57.1 \%(8 / 14)$ & \\
\hline Agree & $34.4 \%(11 / 32)$ & $33.3 \%(6 / 18)$ & $35.7 \%(5 / 14)$ & \\
\hline Neutral & $12.5 \%(4 / 32)$ & $22.2 \%(4 / 18)$ & $0 \%$ & \\
\hline Disagree & $3.1 \%(1 / 32)$ & $0 \%$ & $7.1 \%(1 / 14)$ & \\
\hline Strongly disagree & $3.1 \%(1 / 32)$ & $5.6 \%(1 / 18)$ & $0 \%$ & \\
\hline Confident that privacy would be protected when using TT app & & & & 0.41 \\
\hline Strongly agree & $36.4 \%(12 / 33)$ & $44.4 \%(8 / 18)$ & $26.7 \%(4 / 15)$ & \\
\hline Agree & $30.3 \%(10 / 33)$ & $16.7 \%(3 / 18)$ & $46.7 \%(7 / 15)$ & \\
\hline Neutral & $21.2 \%(7 / 33)$ & $27.8 \%(5 / 18)$ & $13.3 \%(2 / 15)$ & \\
\hline Disagree & $6.1 \%(2 / 33)$ & $5.6 \%(1 / 18)$ & $6.7 \%(1 / 15)$ & \\
\hline Strongly disagree & $6.1 \%(2 / 33)$ & $5.6 \%(1 / 18)$ & $6.7 \%(1 / 15)$ & \\
\hline
\end{tabular}

\footnotetext{
${ }^{\dagger}$, Fisher's exact test; ${ }^{\ddagger}$, one person did not provide answer; other $\mathrm{P}$ values calculated using Chi-Square testing. TT, Tension Tamer.
} 
Table 4 Exemplar quotes and connected themes

\begin{tabular}{|c|c|}
\hline Themes & Exemplar quotes \\
\hline \multicolumn{2}{|l|}{ Theme I: stress was pervasive } \\
\hline $\begin{array}{l}\text { Type of stress: on being } \\
\text { overworked/too many } \\
\text { responsibilities }\end{array}$ & $\begin{array}{l}\text { "I am a single man, four boys, so I work two jobs, I go to school full time, that is just stressful } \\
\text { by itself trying to make time for work, make time for school, make time for my kids, they all play } \\
\text { sports." }\end{array}$ \\
\hline $\begin{array}{l}\text { Type of stress: on financial issues/ } \\
\text { unsafe living environment }\end{array}$ & $\begin{array}{l}\text { "I live some place that I really can't afford but, I don't want to move anywhere else because I have } \\
\text { boys, and places that I can afford has high crime activity, so, everything is stressful to me." }\end{array}$ \\
\hline $\begin{array}{l}\text { Type of stress: on overworked/ } \\
\text { caretaking }\end{array}$ & $\begin{array}{l}\text { "I am a mother of two, and my husband is disabled, and has been out of work due to his medical } \\
\text { issues, and I'm trying to juggle three jobs." }\end{array}$ \\
\hline $\begin{array}{l}\text { Type of stress: on job stresses/ } \\
\text { living environment }\end{array}$ & $\begin{array}{l}\text { "I have to look for a new job. Probably next year early, so that's stressful, but it's manageable. I'm } \\
\text { doing some renovations in my home, and it's turned upside down, and that stresses me out a } \\
\text { whole lot." }\end{array}$ \\
\hline $\begin{array}{l}\text { Health effects of stress: on taking } \\
\text { BP medication }\end{array}$ & $\begin{array}{l}\text { "I was prescribed, but I refused to take it, (laughs), it runs in my family, and I don't want to ..., } \\
\text { everybody who gets on it doesn't get off... and I didn't want that, so, I just didn't take it, and, I just } \\
\text { start pray a little hard, and it's been okay, lately." }\end{array}$ \\
\hline $\begin{array}{l}\text { Health effects of stress: on rapid } \\
\text { heart rate }\end{array}$ & $\begin{array}{l}\text { "I have my moments when it's really, really high (referring to heart rate), and I'll just take a break, lay } \\
\text { down, you know, calm down, and it's, it's okay, usually, but it was, it was during a time when I was } \\
\text { really stressed." }\end{array}$ \\
\hline $\begin{array}{l}\text { Health effects of stress: on } \\
\text { overeating/weight gain }\end{array}$ & $\begin{array}{l}\text { "I am stressed, okay, so I am eating a little bit more, I'm gaining more weight, my blood pressure is } \\
\text { higher, you know." }\end{array}$ \\
\hline $\begin{array}{l}\text { Health effects of stress: on high } \\
\text { blood sugar }\end{array}$ & $\begin{array}{l}\text { "Doctor told me that I was showing early signs of diabetes, so you got to get a hold on this, but } \\
\text { now I am stressed, eating, doing other things that were leading to it." }\end{array}$ \\
\hline $\begin{array}{l}\text { Health effects of stress: on BP and } \\
\text { becoming flush }\end{array}$ & $\begin{array}{l}\text { "I'm not sure of the correlation but I know when I get very stressful I feel flushed, assuming that my } \\
\text { blood pressures go up, my face starts getting warm." }\end{array}$ \\
\hline \multicolumn{2}{|c|}{ Theme II: coping strategies: both positive and negative } \\
\hline On using prayer to cope & $\begin{array}{l}\text { "It could be at work, in the bathroom stall where it's quiet. Especially at home, in my bedroom at } \\
\text { night, sometimes I wake up at three in the morning and l'll say a prayer." }\end{array}$ \\
\hline On using exercise/music & "Exercise, listening to music; Jazz or something like that." \\
\hline On using exercise & "Walking. A walk is very good for when you are stressful. It gives you a chance to clear your mind." \\
\hline $\begin{array}{l}\text { On negative coping by isolating } \\
\text { one's self }\end{array}$ & $\begin{array}{l}\text { "I think that for me, I withdraw. Yeah, I definitely isolate myself because I don't want to be a } \\
\text { problem to anybody else. And I'm always like, let me figure this out." }\end{array}$ \\
\hline
\end{tabular}

Theme III: BAM training was easy to incorporate (and noted recommendations)

Referring to quick start instructions "As easily accessible as possible. On one of the front pages: (it should read) 'Click here to watch to add video instructions instructional video. That is where the YouTube comes in, something you can go on-line to hear further instructions."

Referring accessing music/sounds into session

"One thing l've liked about those types of tapes or CD's that I've listened to in the past; some of them actually have some kind of nature sound in the background, like waves."

Referring to options using guided sessions

"I think the key is it needs to help you meditate, I mean put my ear buds in and I start the process, and I need to be able to close my eyes and it give me audible responses; what she was saying. If it's just meditate, I don't need a quick start any more, I can meditate." (describing the desire for additional content to guide meditation)

On customization and personalization of app

"Well another thing is that I would like to use my own pictures as my background. So you can have an option where you can go to your own photos. And like pick a picture of a sunset you took. Or like, your dog." (noting customization needs)

Table 4 (continued) 
Table 4 (continued)

\begin{tabular}{ll}
\hline Themes & Exemplar quotes \\
\hline Theme IV: tracking stress responses with a metric was useful \\
$\begin{array}{ll}\text { Relating usefulness of prior health } \\
\text { apps tracking metrics }\end{array}$ & $\begin{array}{l}\text { "Yeah and I had that move app (describing another smartphone app) that did the same things, it's } \\
\text { like, how many steps did I make? And I would look back, two days or three days; I made like } 5000\end{array}$ \\
$\begin{array}{l}\text { On feedback of the HR chart after } \\
\text { demo session }\end{array}$ & $\begin{array}{l}\text { "The graph, it's useful but it could be defined a bit more, 'cause it was like "you had a forty drop. (40 } \\
\text { beats per min HR drop) We don't see it. If you could put a red mark, this was your highest this was } \\
\text { the drop that we were talking about." } \\
\text { "If your doctors have access to your information there will be a way that we can say, Dr. such and } \\
\text { physicians }\end{array}$ \\
\hline
\end{tabular}

$\mathrm{BP}$, blood pressure; BAM, breathing awareness meditation.

\section{(ii) Subtheme: health effects of stress}

Many of the stressors reflected personal medical conditions and were a concern to the participants. Participants showed varying levels of control of their stress and while some responded with humor or avoidance, others noted that physical and emotional reactions to stress additionally contributed to existing stress levels. Some participants showed concern and alarm when describing their physical reactions when they become stressed that border on the symptomology of panic attacks (i.e., increased anxiety, insomnia, feeling hot, feel their heart pounding, etc.). Several were noted dealing with their stress by eating more, which has affected their weight status causing a cyclical effect resulting in more stress.

\section{Theme II: coping strategies were both positive and negative}

Most of the participants were able to relate their adaptive coping mechanisms, both positive and negative reactions, used in handling their stressors. Positive examples included exercise, faith and prayer, staying organized, humor, talking with friends, and finding quiet time to self-soothe, whereas negative examples included overeating, smoking, excessive alcohol intake.

\section{Theme III: BAM training was easy to incorporate}

Participants responded positively to the TT app demonstration and made suggestions concerning Quick Start instructions and the general use of the app. These included incorporation of an easily accessible introductory instructional video, addition of relaxing background audio (e.g., nature sounds- rainfall, ocean waves lapping on shore, etc.), and the option to select relaxing images for the background including downloading favorite scenesseashore, favorite pet waiting for a walk, etc. They recommended a quick tutorial but also provide in-depth instructions to troubleshoot usage issues. They noted that the app should be intuitive to use should someone wish to skip any training. They noted that any delay in getting the app to initialize or work correctly would be met with consequential disuse, citing individual prior experience with other apps. Customization and personalization were frequently implied in their responses. There was also an interest in continuing new content so the experience of using an app remains interesting.

\section{Theme IV: tracking stress responses with a metric was useful}

Overall, there was enthusiasm for the potential to monitor HR and subsequently reduce BP through the TT app, including comparisons showing improvement over time. Many were uncertain on the best method to communicate session results, though the HR response graph was favorably mentioned because it showed their individual response and provided feedback they were using the app correctly. There were requests on how to share their results with a personal physician or others. Participants related the usefulness of the app's HR data to that of other mHealth apps such as fitness trackers.

Combining and comparing quantitative and qualitative reports, we found agreement on all data sources except the African American groups reported higher stress. Therefore, results support that culturally tailoring BAM smartphone 
apps is not warranted based from this sample. Data was combined to describe the overall results. Overall, the results showed agreement that BAM apps was an acceptable option to treat stress that held no confidentiality or trust issues. Participants knew that high BP places individuals at increased risk for disease but most were unaware of their own BP status and category of risk. Participants were savvy about use of smartphones, felt the TT app was easily learned and was easy to use. However, there was a disconnect with participant knowledge about how HR was associated with BP. Participants expressed desire for additional visual feedback about HR reductions (e.g., comparison and summary charts by week, month, etc.) and were excited about the potential to use this app to reduce their stress.

\section{Discussion}

This study investigated the acceptability of a BAM mHealth app designed to reduce stress and assist in BP control for White and African American preEH populations. Low cost, widely disseminable strategies including smartphoneenabled BAM approaches may be a viable option for practitioners to recommend to patients. BAM may augment other strategies for BP management including other nonpharmacological approaches including the DASH diet, exercise for weight loss, reduced sodium intake, increasing physical activity, and moderation of alcohol intake (51). The uptake in health behavior programs may be mediated by culturally tailored content which is warranted for most behavioral interventions. Consideration of potential cultural differences is particularly important in mHealth research given long-standing concerns related to trust in the research and health care systems that may influence use of mHealth tools or adherence to mHealth research procedures. However, in this sample, there were no issues in trust and no other statistically significant or qualitative differences between Whites and African Americans were found besides the level of stress. This may imply homogeneity in this sample when considering access and intervention preferences using mHealth technology. A notable knowledge gap in our sample was the understanding of EH risk categories. This suggests BAM apps may need to include education about EH risk strata. Lastly, participants were acceptable concerning privacy issues with using or sharing health information from the TT app with providers in addition to other mHealth smartphone apps which warrants further consideration linking app use and provider utilization.

Two of the four main themes from the qualitative analysis focused upon stress: (I) stress was pervasive and (II) coping strategies were both positive and negative. We found that participants had many issues and situations in their lives that resulted in chronic stress exposure which resulted in both positive and maladaptive coping styles. These maladaptive coping styles have long been noted in stress research to further contribute to perceived stress, as well as development or exacerbation of various chronic diseases (e.g., EH, obesity, CVD, stroke, certain cancers, etc.) $(3,17)$. There were, however some positive coping strategies noted including taking a "time out," relaxing and exercising. From the results of this study, it is suggested that many though the TT app or BAM programs could belong as a positive coping strategy to help them relax and adapt to stressful circumstances in their lives.

The focus groups also resulted in two additional themes relating to the mechanics and outputs of using the TT app: (III) BAM training was easy to incorporate; and (IV) tracking stress responses was useful. In theme III, participants wanted to see value in the app immediately. If the app was slow to work, hard to understand or confusing, the participants noted they would become frustrated and want to exit the app and try something else, which would affect sustainability of a BAM program. This is a particularly important consideration for mHealth apps designed to deliver repeated sessions of an intervention, such as TT. The participants' input led to an important focus concerning Quick Start guides and how they are used to educate first-time users on proper operation of app features. Participants suggested incorporation of visual depictions of use scenarios, help or tutorial videos and limited text. Generalization of this theme to other mHealth apps may be important when considering sustained use.

With regard to theme (IV) tracking stress responses were useful, the tracking history of sessions and HR results were deemed as a critical feature. Participants wanted to be able to readily see changes in tracked metrics over time (e.g., average HR, absolute HR reduction per session, etc.) and be able to share these with their provider if they desired. There were discussions on being able to view cumulative reports of the metrics automatically over time (e.g., weeks, months) but needs were dependent on the respondent. When discussing features, participants wanted continual content updates (e.g., additional guided sessions, enhanced reporting, etc.) and be able to load in their own media (e.g., pictures of calming scenes, or their own music or peaceful 
sounds during the meditation session). These align but transcend the theories of human computer interaction and the behavioral implications of the technology acceptance model, which mostly align to delivering a static product (52-54). Whereas those models allow input and design parameters to be influenced by the target population for an end product, a different approach is postulated here: that the end product should be more dynamic or fluid in nature, or allow individual customization. Additional configurations through a settings menu may be warranted to allow personalization to the look, outputs, and feel of mHealth app to more closely attend to the individual preferences and needs of the user. These customizations may drive an intimate ownership of the app and may increase likelihood of sustained use. Caution should be used when considering adding too many features or customization as they may confuse users with too much clutter in the interface. The utility of needing customization may be moderated by the level of comfort and experience one may have using technology, and specifically familiarity with other smartphone apps.

Our findings from this study carry implications beyond the demonstrated app though are not without limitations. The generalizability of our results may be limited to African American and White adults who have at least a high school education, are employed and with known preEH. Our sample also had higher smartphone utilization rates compared to the general public $(75.8 \%$ vs $68 \%)$ which may be influenced by education and socioeconomic status (55). Generalization of our findings may also be more applicable to other preEHs, and likely EHs, who are motivated to uptake healthy behaviors. Due to the fact that the study participants responded and took part in a health-related focus group, they may be more educated about health topics and smartphone apps than others with preEH who did not participate. Thus, our findings may over-represent potential interest and acceptability of smartphone BAM apps. Our study may also be limited due to the relatively smaller number of focus groups, though qualitative saturation was met and groups were combined to report on the overall results.

This study may also be applicable to a wider range of populations including those with stress, anxiety or addiction issues and may provide relief by learning and practicing BAM. The lack of findings to indicate a need for a culturally tailored approach was somewhat surprising. Many health behavior programs have touted the need and integration of culturally appropriate frameworks compared to usual care and though results have varied, study outcomes lean toward a positive effect (31). Culturally tailoring interventions are recommended to reduce health disparities (28), though our results, cognizant of our limited sample, do not support this need in designing BAM apps. This may imply that BAM app design is more aligned with an individual approach such as been found in other individually tailored web-based programs (56). Culturally tailored needs for other racial or ethnic groups, especially if the app is to be translated into a different language or tailored to motivating elements in a specific culture (e.g., religious beliefs, national movement, etc.) may have different results. The decision in which to employ an individually tailored or culturally tailored approach for smartphone apps needs further exploring across various population samples, types of programs, and differing mHealth behavior topics.

In summary, this study's preEH participants responded favorably to the concept of using a smartphone-based BAM program to manage their stress. Moreover, the overwhelming majority felt the TT app could aid them as a coping mechanism by replacing maladaptive strategies with BAM to reduce their stress. These results suggested the TT app as well as other mHealth apps should refine their software development approach to incorporate the options to personalize the user-interface and include metric reporting in order to drive use. Adherence may be augmented by such design parameters where customization of the app's look and feel drives ownership that adds to the appeal for continued use. Although the need for cultural tailoring BAM app designs for White or African American participants was not found, our findings do not imply that culturally tailored program are unnecessary with regard to other mHealth approaches, though in the case of BAM an individual tailoring approach, it may be unnecessary.

\section{Acknowledgements}

Funding: This work was supported by funding from the National Institutes of Health's National Heart, Lung, and Blood Institute (R01 HL114957 to FA Treiber), the National Institute of Mental Health (K23 DA038257 to ZW Adams) and the South Carolina Clinical and Translational Research Institute, with an academic home at Medical University of South Carolina, through the National Institute of Health (grant number UL1 TR000062). The content does not represent the official views of the National 
Institute of Health.

\section{Footnote}

Conflicts of Interest: The authors have no conflicts of interest to declare.

Ethnical Statement: The study was approved by the Medical University of South Carolina Ethical Review Board (No. Pro00021312). Written informed consent was obtained from all participants for publication of this manuscript and any accompanying images.

\section{References}

1. Holmes CS, Chen R, Streisand R, et al. Predictors of youth diabetes care behaviors and metabolic control: a structural equation modeling approach. J Pediatr Psychol 2006;31:770-84.

2. Kubzansky LD, Davidson KW, Rozanski A. The clinical impact of negative psychological states: expanding the spectrum of risk for coronary artery disease. Psychosom Med 2005;67 Suppl 1:S10-4.

3. Rozanski A, Blumenthal JA, Davidson KW, et al. The epidemiology, pathophysiology, and management of psychosocial risk factors in cardiac practice: the emerging field of behavioral cardiology. J Am Coll Cardiol 2005;45:637-51.

4. Williams DR, Neighbors HW, Jackson JS. Racial/ethnic discrimination and health: findings from community studies. Am J Public Health 2003;93:200-8.

5. Dressler WW. Social and Cultural Dimensions of Hypertension in Blacks: Underlying Mechanisms. In: Fray JC, Douglas JG. editors. Pathophysiology of Hypertension in Blacks. New York, NY: Oxford University Press, 1993:69-89.

6. Kaplan MS, Nunes A. The psychosocial determinants of hypertension. Nutr Metab Cardiovasc Dis 2003;13:52-9.

7. Lovallo WR. Stress and Health: Biological and Psychological Interactions. 2nd ed. Thousdand Oaks, CA: Sage Publications, 2005.

8. Greenberg A, Berktold J. APA Survey Shows Holiday Stress Putting Women's Health at Risk. PR Newswire, a UBM plc company: American Psychological Association, 2006.

9. Bentley B, De Jong MJ, Moser DK, et al. Factors related to nonadherence to low sodium diet recommendations in heart failure patients. Eur J Cardiovasc Nurs
2005;4:331-6.

10. Elder CR, Gullion CM, Funk KL, et al. Impact of sleep, screen time, depression and stress on weight change in the intensive weight loss phase of the LIFE study. Int J Obes (Lond) 2012;36:86-92.

11. Daubenmier JJ, Weidner G, Sumner MD, et al. The contribution of changes in diet, exercise, and stress management to changes in coronary risk in women and men in the multisite cardiac lifestyle intervention program. Ann Behav Med 2007;33:57-68.

12. Rutters F, Nieuwenhuizen AG, Lemmens SG, et al. Acute stress-related changes in eating in the absence of hunger. Obesity (Silver Spring) 2009;17:72-7.

13. Dallman MF. Stress-induced obesity and the emotional nervous system. Trends Endocrinol Metab 2010;21:159-65.

14. Kuhl EA, Fauerbach JA, Bush DE, et al. Relation of anxiety and adherence to risk-reducing recommendations following myocardial infarction. Am J Cardiol 2009;103:1629-34.

15. Gregoski MJ, Barnes VA, Tingen MS, et al. Differential Impact of Stress Reduction Programs upon Ambulatory Blood Pressure among African American Adolescents: Influences of Endothelin-1 Gene and Chronic Stress Exposure. Int J Hypertens 2012;2012:510291.

16. Meyer PA, Yoon PW, Kaufmann RB, et al. Introduction: CDC Health Disparities and Inequalities Report - United States, 2013. MMWR Suppl 2013;62:3-5.

17. Rainforth MV, Schneider RH, Nidich SI, et al. Stress reduction programs in patients with elevated blood pressure: a systematic review and meta-analysis. Curr Hypertens Rep 2007;9:520-8.

18. Black DS, Milam J, Sussman S. Sitting-meditation interventions among youth: a review of treatment efficacy. Pediatrics 2009;124:e532-41.

19. Barnes VA, Pendergrast RA, Harshfield GA, et al. Impact of breathing awareness meditation on ambulatory blood pressure and sodium handling in prehypertensive African American adolescents. Ethn Dis 2008;18:1-5.

20. Barnes VA, Treiber FA, Johnson MH. Impact of transcendental meditation on ambulatory blood pressure in African-American adolescents. Am J Hypertens 2004;17:366-9.

21. Gregoski MJ, Barnes VA, Tingen MS, et al. Breathing awareness meditation and LifeSkills Training programs influence upon ambulatory blood pressure and sodium excretion among African American adolescents. J Adolesc Health 2011;48:59-64. 
22. Brown WL, Gregoski MJ, Tingen MS, et al. Impact of stress reduction interventions on hostility and ambulatory systolic blood pressure in African American adolescents. J Black Psychol 2010;37:210-33.

23. Barnes VA, Gregoski MJ, Tingen MS, et al. Influences of Family Environment and Meditation Efficacy on Hemodynamic Function among African American Adolescents. J Complement Integr Med 2010;7.

24. Ospina MB, Bond K, Karkhaneh M, et al. Meditation practices for health: state of the research. Evid Rep Technol Assess (Full Rep) 2007;(155):1-263.

25. Nidich SI, Rainforth MV, Haaga DA, et al. A randomized controlled trial on effects of the Transcendental Meditation program on blood pressure, psychological distress, and coping in young adults. Am J Hypertens 2009;22:1326-31.

26. Caspi O, Burleson KO. Methodological challenges in meditation research. Adv Mind Body Med 2005;21:4-11.

27. Handel MJ. mHealth (mobile health)-using Apps for health and wellness. Explore (NY) 2011;7:256-61.

28. Barrera M Jr, Castro FG, Strycker LA, et al. Cultural adaptations of behavioral health interventions: a progress report. J Consult Clin Psychol 2013;81:196-205.

29. Fisher TL, Burnet DL, Huang ES, et al. Cultural leverage: interventions using culture to narrow racial disparities in health care. Med Care Res Rev 2007;64:243S-82S.

30. Nierkens V, Hartman MA, Nicolaou M, et al. Effectiveness of cultural adaptations of interventions aimed at smoking cessation, diet, and/or physical activity in ethnic minorities. a systematic review. PLoS One 2013;8:e73373.

31. Hawthorne K, Robles Y, Cannings-John R, et al. Culturally appropriate health education for Type 2 diabetes in ethnic minority groups: a systematic and narrative review of randomized controlled trials. Diabet Med 2010;27:613-23.

32. Tufts KA, Johnson KF, Shepherd JG, et al. Novel interventions for HIV self-management in African American women: a systematic review of mHealth interventions. J Assoc Nurses AIDS Care 2015;26:139-50.

33. McGillicuddy JW, Weiland AK, Frenzel RM, et al. Patient attitudes toward mobile phone-based health monitoring: questionnaire study among kidney transplant recipients. J Med Internet Res 2013;15:e6.

34. Price M, Williamson D, McCandless R, et al. Hispanic migrant farm workers' attitudes toward mobile phonebased telehealth for management of chronic health conditions. J Med Internet Res 2013;15:e76.

35. Ovbiagele B, Jenkins C, Patel S, et al. Mobile health medication adherence and blood pressure control in recent stroke patients. J Neurol Sci 2015;358:535-7.

36. Tong A, Sainsbury P, Craig J. Consolidated criteria for reporting qualitative research (COREQ): a 32-item checklist for interviews and focus groups. Int J Qual Health Care 2007;19:349-57.

37. Gregoski MJ, Vertegel A, Shaporev A, et al. Tension Tamer: delivering meditation with objective heart rate acquisition for adherence monitoring using a smart phone platform. J Altern Complement Med 2013;19:17-9.

38. Mani M, Kavanagh DJ, Hides L, et al. Review and Evaluation of Mindfulness-Based iPhone Apps. JMIR Mhealth Uhealth 2015;3:e82.

39. Gregoski MJ, Mueller M, Vertegel A, et al. Development and validation of a smartphone heart rate acquisition application for health promotion and wellness telehealth applications. Int J Telemed Appl 2012;2012:696324.

40. Gregoski MJ, Vertegel A, Treiber FA. Photoplethysmograph (PPG) derived heart rate acquisition using an Android smart phone. Proceedings of the 2nd Conference on Wireless Health. San Diego, California, October 10-13, 2011.

41. Adams Z, Favela A, Patel S, et al. Breathing meditation smartphone app: Initial findings of dose response trail upon blood pressure (BP) changes among prehypertensive adults. Psychosomatic Medicine 2015;2015;77(3).

42. Gregoski MJ, Sieverdes JC, Brunner Jackson B, et al. Smartphone Breathing Meditation Application Proof of Concept: Linkage Between Nighttime Blood Pressure Reduction and Salivary Alpha-Amylase Awakening Response. Ann Behav Med 2013;45:s240.

43. Vasan RS, Larson MG, Leip EP, et al. Assessment of frequency of progression to hypertension in nonhypertensive participants in the Framingham Heart Study: a cohort study. Lancet 2001;358:1682-6.

44. Cohen S, Williamson GM. The Social Psychology of Health. Oskamp SS. editor. Newbury Park, CA: Sage, 1988.

45. Fink A. How to Design Survey Studies. The Survey Kit. 2nd ed. Thousand Oaks: Sage, 2003.

46. Davis FD. Perceived Usefulness, perceived Ease of Use, and User Acceptance of Information Technology. MIS Quart 1989;13:319-40.

47. Ryan RM, Deci EL. Self-determination theory and the facilitation of intrinsic motivation, social development, and well-being. Am Psychol 2000;5 5:68-78.

48. Riley WT, Rivera DE, Atienza AA, et al. Health behavior models in the age of mobile interventions: are our theories up to the task? Transl Behav Med 2011;1:53-71.

49. LeRouge C, Ma J, Sneha S, et al. User profiles and 
personas in the design and development of consumer health technologies. Int J Med Inform 2013;82:e251-68.

50. Borkan JM. Immersion/Crystallization. In: Crabtree BF, Miller WL. editors. Doing Qualitative Research. Newbury Park: Sage Publications, 1999:179-94.

51. Svetkey LP. Management of prehypertension. Hypertension 2005;45:1056-61.

52. Legris P, Ingham J, Collerette P. Why do people use information technology? a critical review of the technology acceptance model. Information \& Management 2003;40:191-204.

53. Davis FD. User acceptance of informaiton technology: system characteristics, user perceptions and behavioral impacts. Int J Man Mach Stud 1993;38:475-87.

54. Ickin S, Wac K, Fiedler M, et al. Factors influencing quality of experience of commonly used mobile applications. IEEE Communications Magazine 2012;50:48-56.

55. Anderson M. Technology Device Ownership: 2015. Pew Research Center. October 29, 2015.

56. Lustria ML, Cortese J, Noar SM, et al. Computer-tailored health interventions delivered over the Web: review and analysis of key components. Patient Educ Couns 2009; 74:156-73.

doi: $10.21037 /$ mhealth.2017.09.04

Cite this article as: Sieverdes JC, Adams ZW, Nemeth L, Brunner-Jackson B, Mueller M, Anderson A, Patel S, Sox L, Treiber FA. Formative evaluation on cultural tailoring breathing awareness meditation smartphone apps to reduce stress and blood pressure. mHealth 2017;3:44. 